\title{
On graph parameters guaranteeing fast sandpile diffusion
}

\author{
Ayush Choure Sundar Vishwanathan \\ Department of Computer Science and Engineering \\ Indian Institute of Technology \\ Bombay, India \\ \{ayush, sundar\}@cse.iitb.ac.in
}

Submitted: Nov 18, 2012; Accepted: Feb 6, 2015; Published: Feb 25, 2015

Mathematics Subject Classifications: $05 \mathrm{C} 81$

\begin{abstract}
The Abelian Sandpile Model (Dhar 1990) is a discrete diffusion process, defined on graphs, which serves as the standard model of self-organized criticality. One is allowed to add sand particles on the nodes of the graph such that each node can stably hold at most some bounded number of particles. The particles flow through the graph as a consequence of surpassing the node capacities, until they reach a special sink node possessing infinite capacity. These simple dynamics give rise to a very interesting Markovian system. The transience class of a sandpile is defined as the maximum number of particles that can be added without making the system recurrent. We identify a small set of key graph properties that guarantee polynomial bounds on transience classes of the sandpile families satisfying them. These properties governing the speed of sandpile diffusion process are volume growth parameters, boundary regularity type properties and non-empty interior type constraints.

This generalizes a previous result by Babai and Gorodezky (2007) in which they establish polynomial bounds on the $n \times n$ grid. Indeed the properties we show are based on ideas extracted from their proof as well as the continuous analogs in the theory of harmonic functions.
\end{abstract}

Keywords: Abelian Sandpile Model; Harmonic functions on graphs; Transience Class Problem of Sandpiles

\section{Introduction}

The Abelian sandpile model(ASM) is a type of discrete diffusion process defined on graphs. The model was pioneered by Dhar [21] while investigating the phenomena of self-organized criticality in the dynamics of sandpile formation. A close cousin is the celebrated looperased random walks model [33]. If one plots the length of a loop-erased random walk 
against time, the results obtained are qualitatively similar to the one obtained from observing sandpile weights against time. Indeed the similarity extends into numerous seemingly different phenomena like stress distribution in earthquakes, size distribution in raindrops, etc (see the recent comprehensive survey article by Dhar [20]).

In the ASM, "sand particles" are added at vertices of a (multi)graph. A site (vertex) is stable as long as the number of particles at the site are less than its degree. Adding any more particles makes the site unstable and is accompanied by the unstable site passing a particle along each incident edge to its neighboring sites. This relaxation process is called toppling. There is a special site called the sink which cannot topple. To ensure that every relaxation process eventually stabilizes, one needs the condition that the sink is reachable from every other site. In the course of evolution via particle additions and toppling, the sandpile goes through a sequence of configurations. The configurations which cannot be revisited are called transient, while those which are reachable from every other configuration are called recurrent. Typically, one starts with the empty configuration and as particles are added, one moves through transient configurations till a recurrent configuration is reached. Thereafter the configurations stay recurrent. The steady state behavior of any Markovian system is characterized by its set of recurrent states. While modeling natural phenomena, the set of recurrent stated is supposed to capture the behavior one is trying to emulate. Intuitively, if the time taken to reach recurrence is too high (e.g. exponential in model size), the identification of long-term behavior with recurrent states becomes doubtful.

The parameter of importance in our discussion will be the number of particles needed to reach recurrence. The case of a random adversary, where particles are added in a uniformly random manner across the graph, yields to a simple coupon collector type argument. This results in polynomial bounds on the expected time to recurrence (as previously noted in [4]). The non-trivial case arises when particles are added adversatively. Here the problem acquires a distinctly potential theoretic flavor. In this scenario, our goal is to add particles so as to avoid a recurrent state for as long as possible. This problem was highlighted by Babai and Toumpakari [4] where they define this number of particles as the transience class of the sandpile. This later motivated the insightful work by Babai and Gorodezky [3] on grid based sandpiles. In this breakthrough paper, the authors show that for the standard $n \times n$ grid based sandpiles, the maximum number of particles one can add before hitting a recurrent state is $O\left(n^{30}\right)$, later improved to $O\left(n^{15}\right)$ [2]. Using arguments based on LP-duality, harmonic functions, and symmetry, Choure and Vishwanathan [11] improve this bound to $O\left(n^{7} \log n\right)$. They also establish a sandwich theorem for the transience class of any sandpile in terms of the values of harmonic functions, establishing a correspondence between random walks and sandpile diffusions.

The relevance of "grid" sandpile comes from the fact that grids serve as the standard discrete substitutes of planar regions in statistical physics. It is therefore natural to ask if other graphs which can arise as regular (or irregular even) tessellations of plane (or higher dimensional spaces) also obey polynomial bounds on their transience classes. More generally, one might not even want to pose restrictions on the integrality of dimension of 
the space from which these graphs arise. Indeed graphs associated with fractals are quite well analyzed in potential theory. We refer the reader to the excellent monograph by Telcs [46] for a succinct yet thorough introduction to literature in this area. Our study is aimed at trying to understand the underlying potential theoretic properties which characterize polynomial transience.

\subsection{Our Contribution}

To state our main result, we outline some basic notions in this section. These definitions will be explained in detail with appropriate context in following sections. The informal definition of a sandpile, and its dynamics are described above. We will call an indexed countably infinite set of sandpiles, a sandpile family. For an instance of sandpile, we will take an undirected connected graph $G$ with a special sink node. The distances in this graph will be defined using the shortest path metric. A ball $B(v, r)$ is the set of all nodes which are at a distance of at most $r$ from $v$. The volume of this ball, denoted by $\operatorname{Vol}(B(v, r))$, is the number of edges in the induced subgraph of the ball. We define the following properties.

Definition 1. Polynomial Volume Growth Property $\left(V_{\alpha}\right)$ : A graph family $\mathcal{S}$ has polynomial volume growth property $\left(V_{\alpha}\right)$ with growth exponent $\alpha$ if there exist constants $\delta$ and $\Delta$ such that for any graph $S_{i} \in \mathcal{S}$ and every node $v \in V\left(S_{i}\right)$, if we consider the $B(v, r)$ around $v$, its volume satisfies the following bounds:

$$
\delta r^{\alpha} \leqslant \operatorname{Vol}(B(v, r)) \leqslant \Delta r^{\alpha}
$$

Definition 2. High Local-Conductance Property $\left(\mathrm{hLC}\left(C_{\sigma}\right)\right)$ : A sandpile family $\mathcal{S}$ satisfies $\mathrm{hLC}\left(C_{\sigma}\right)$ if there exists a constant $C_{\sigma}$, independent of the index $i$ of the sandpile $S_{i} \in \mathcal{S}$, such that for any site $v \in V\left(S_{i}\right)$ and any ball $B(v, r)$ in $S_{i}$, placing $C_{\sigma} \cdot \operatorname{Vol}(B(v, r))$ particles at $v$ allows every site in the ball $B(v, r)$ to receive at least one particle.

We will be needing the definition of harmonic functions for describing the next property. Given a connected graph $G$ and a function $\pi: V(G) \rightarrow \mathbb{R}$, we say that $\pi$ is harmonic over the vertex set $V_{h}$ if,

$$
\frac{1}{\operatorname{degree}(v)} \sum_{u \sim v} \pi(u)=\pi(v) \quad v \in V_{h}
$$

Using this, we define the mean value property over graphs.

Definition 3. Mean Value Property $\left(\operatorname{MV}\left(C_{h}\right)\right)$ : A sandpile family $\mathcal{S}$ satisfies $\operatorname{MV}\left(C_{h}\right)$ if there exists a constant $C_{h}$, independent of the index $i$ of the sandpile $S_{i} \in \mathcal{S}$, such that for any graph $S_{i}=\left(V_{h} \cup\{s\}, E_{h} \cup \delta_{E} V_{h}\right)$ if $\pi: V\left(S_{i}\right) \rightarrow[0,1]$ is a function harmonic over the ball $B(v, r) \subset V_{h}$, the following inequality holds,

$$
\sum_{u \in B(v, r)} \pi(u) \geqslant C_{h} \pi(v) \operatorname{Vol}(B(v, r))
$$


Consider a sandpile family $\mathcal{S}$ and a member graph $S_{i}$. Call a path $P=v_{1}, \ldots, v_{k}$ from $v_{1}$ to $v_{k}(1, l)$-central if $\operatorname{dist}\left(v_{i}, \operatorname{sink}\right)$ varies linearly with $i$, or if its length is bounded by $l \log \left(\left|S_{i}\right|\right)$. We call it $(k, l)$-central if it is a juxtaposition of at most $k$ central paths, where $k$ and $l$ are constants independent of graph index (and size). The concept of linearly varying distance functions is discussed with examples in later sections.

Definition 4. Non-empty Interior Property $(\mathrm{NI}(k, l))$ : A sandpile family $\mathcal{S}$ has nonempty interior property, $\mathrm{NI}(k, l)$, if for every member sandpile graph $S_{i} \in \mathcal{S}$ and if for every pair of vertices $v, w \in V\left(S_{i}\right)$, there exists a path between them which is $(k, l)$-central. Here, $k$ and $l$ are constants independent of the sandpile index $i$.

Our main result is the Epicenter Propagation Theorem, which establishes polynomial bounds on the maximum number of particles that can be added to a sandpile before making it recurrent.

Theorem 1.1. (Epicenter Propagation Theorem) Given a sandpile family $\mathcal{S}$ which satisfies $h L C\left(C_{\sigma}\right), V_{\alpha}, M V\left(C_{h}\right)$, and $N I(k, l)$, then for any member sandpile $S_{i} \in \mathcal{S}$, the transience class satisfies the following bounds

$$
\operatorname{tcl}\left(S_{i}\right) \leqslant C_{\sigma} \Delta n^{k+(2 l+1) k \log _{\hat{g}}\left(\frac{C_{\sigma}}{C_{h}} \frac{\Delta(\Delta+1)}{\delta} 3^{\alpha}\right)}
$$

Here, $n=\left|S_{i}\right|$.

To prove our main result, we first show that if the graph has uniform polynomial volume growth, satisfies mean value property, and has high local conductance of particle percolation, it satisfies a fundamental superposition property, which is the discrete analogue of the celebrated superposition principal. This property allows one to place particles at a suitable set of multiple nodes and observe the same potential response at a particular site, as one would have by placing all these particles at one site. Using this we prove one of the main results in this paper, the single step Epicenter Propagation Lemma. This is the generalization of the traveling diamond lemma proved in Babai and Gorodezky [3]. Finally, using the single step version and the non-empty interior property, we derive the general Epicenter Propagation theorem which demonstrates effective polynomial bounds on the transience class of large class of sandpile graphs. This result forms the graph theoretic analogue of the classical Harnack's inequality and enables one to prove general bounds on harmonic functions without resorting to limiting arguments linking random walks on graphs to Brownian motions on Euclidean spaces. We conclude with a discussion on open problems which form the logical next step of this work.

\subsection{Related Work}

Random walks and Sandpile: The connections between sandpiles and random walks have been discussed under various contexts. Dhar [20], for example, summarizes the connection between the Loop Erased Random Walks (LERW) and sandpiles. We refer the reader to the excellent text by Lawler and Limic [33] for an introduction to basic 
properties of LERW. The connection with simple random walks is more intuitive and reveals a natural analogy between properties of sandpiles and electric networks. Choure and Vishwanathan [10] discuss a reduction of the transience class computation problem to that of estimating potentials in a related electric circuit. These connections are not surprising as the combinatorial Laplacian of the underlying graph of the sandpile, acts as a kind of generator for both the models. For an introduction to these potential functions, see Bollobás [9]. For a discussion on estimating these potentials on graphs coming from geometric settings, see [46]. Levine and Peres [34] discuss a potential theoretic approach to analyzing the rotor router model, the divisible sandpile and other related models. See [35] for a discussion on the spherical asymptotics of diffusions on lattice graphs. One of the key questions they answer is that, when diffusion starts from some point, then the distances from starting point to the farthest and to the nearest boundary point of the hit region, differ by some multiplicative constant. Boundary dissipations do not matter in this scenario. In our present work, we analyze the transience class problem in the setting of bounded graphs. Indeed, under the other assumptions we make about the graph families, it is the presence of a sink node that makes the problem interesting and non-trivial. Our results show that the dissipation of sand particles through the boundary is limited by a polynomial. Consequently, the entire sandpile is flooded in polynomially many particle additions, regardless of addition strategy.

Diffusions and Potential Theory on Graphs: Delmotte [19] shows how the Harnack's inequality over graphs leads to non-trivial results on heat diffusions. They bound the growth of harmonic functions using Gaussian estimates of the heat kernel, all of which follows from the assumption that the graph under consideration follows a parabolic Harnack's inequality. Chung and Yau [14] derive the Harnack inequality for certain degree bound graphs. The inequality is then used to bound the graphs' Neumann eigenvalues. In [17], they derive Harnack's inequality of Abelian homogenous graphs. Furthermore, Chung and Yau [16] derive lower bounds on log-Sobolev constants by establishing logHarnack inequalities on graphs. Bounding the log-Sobolev constants is important as it helps in establishing convergence bounds on random walks on graphs, see Diaconis and Saloff-Coste [23]. Chung and Yau [15], derive bounds on the eigenvalues of the Laplacians using the Sobolev inequalities and heat kernel estimates.

Electric Networks: The classical theory of random walks [24, 36, 37] has some very powerful and intuitive results which have recently found widespread application in theoretical computer science. Christiano et. al. [13] discuss the fastest known algorithm for computing approximate maximum $s-t$ flows in capacitated undirected graphs. Their algorithm constructs the approximate flows by essentially using the electric current flows on the same network with $s$ and $t$ as poles. Earlier, Kelner et. al. [29] used arguments based on random walks to formulate the fastest known algorithm for generating spanning trees from the uniform distribution. Spielman and Srivastava [43] construct good sparsifiers of weighted graphs via an efficient algorithm for computing approximate effective resistance between any two vertices. Indeed, a deeper understanding of harmonic functions is as 
much of interest to a computer scientist as to a potential theorist and the benefit of this confluence has been mutual. A significant example is the seminal work by Arora, Rao and Vazirani [1] on embeddings of negative type which give an $O(\sqrt{\log n})$ approximation algorithm for computing graph conductance.

Other Results in Sandpiles: As already mentioned, research problems in the Abelian sandpile model span across numerous areas. We make a passing mention to some of this work. Notable advances in complexity theoretic flavor include proof of the onedimensional sandpiles prediction problem in LOGDCFL by Miltersen [39]. Schulz [41] mentions a related NP-complete problem. The group structure of the space of recurrent configurations, first introduced by Dhar et. al. [22] is also considered a fertile area of analysis. Cori and Rossin [18] show that sandpile groups of dual planar graphs are isomorphic. Toumpakari [48] discusses some interesting properties of sandpile groups of regular trees. Questions related to group rank are studied in particular, the paper is concluded with an interesting conjecture on the rank of all Sylow subgroups of the sandpile group. Specific families of graphs like square cycles $C_{n}^{2}, K_{3} \times C_{n}, 3 \times n$ twisted bracelets, etc have been analyzed. We refer the reader to [26, 27, 42].

\section{Basic Properties of The Abelian Sandpile Model}

Our notation and terminology follows Babai and Gorodezky [3].

Definition 5. A graph $G$ is an ordered pair $(V(G), E(G))$ where $V(G)$ is called the set of vertices and $E(G)$ is a multiset of 2 -subsets of $V$, called the set of edges.

The degree of a vertex $v \in V$ is defined as the number of edges in $E$ which contain $v$. Two vertices $v$ and $u$ are called adjacent (or neighboring) if $(u, v) \in E$. A path between two vertices $u$ and $v$ is an ordered sequence of edges $e_{1}, e_{2}, \ldots, e_{k}$ such that $u \in e_{1}, v \in e_{k}$ and for all values of $i, e_{i} \cap e_{i+1} \neq \phi$. The graph $G$ is connected if there exists a path between any pair of vertices.

For an instance of Abelian Sandpile Model, we take a connected graph $G$ with a special vertex called the sink, denoted $s \in V$. Non-sink vertices in $G$ are called ordinary vertices and this set will be denoted by $V_{o}=V-\{s\}$.

Definition 6. The configuration $c$ over a sandpile $G$ is defined as a map $c: V_{o} \rightarrow \mathbb{Z}^{+}$. It will be represented as a vector. The weight of $c$ is $|c|=\sum_{v \in V_{o}} c(v)$.

The configuration $c$ tells us the number of sand particles that each of the ordinary sites currently contain. The empty configuration is the zero vector. The capacity of a site is the maximum number of particles that it can hold and is one less then the degree of the node.

Definition 7. An ordinary node $v$ is said to be unstable in a configuration $c$ if $c(v) \geqslant$ degree $(v)$. If all the sites in a configuration stable, the configuration is stable, else it is referred to as unstable. 
When a site is unstable it is said to topple, i.e. pass on some of its particles to its neighbors. When a site $v$ topples once, it loses degree $(v)$ particles and each neighbor of $v$ acquires a particle for every edge common with $v$. The sink node, by definition, never topples. We start with the empty configuration and keep adding particles one by one on sites of our choice and topple when necessary.

The ASM evolves in time through two modes, particle addition at sites and relaxation of unstable sites via topplings. A toppling sequence is an ordered set of configurations where every configuration can be obtained from the previous one by toppling some site unstable in it. The case of many sites becoming unstable simultaneously also poses no complication as the order in which they are subsequently relaxed does not effect the final stable configuration that is obtained at the end of toppling sequence, hence the prefix abelian. Elementary proofs of such confluence properties can be found in the pioneering paper on ASMs by Dhar [21].

Notation: We write $c_{1} \geqslant c_{2}$ if $\forall v, c_{1}(v) \geqslant c_{2}(v)$, and $c_{1} \vdash c_{2}$ if there is a toppling sequence which takes $c_{1}$ to $c_{2}$. Lastly we write, $c_{1} \rightarrow c_{2}$ if $\exists c_{3} \geqslant c_{1}$ such that $c_{3} \vdash c_{2}$. We say that a configuration $c_{2}$ is reachable from $c_{1}$ if $c_{1} \rightarrow c_{2}$ and unreachable otherwise. In words, one can add particles to certain sites in $c_{1}$ so that there exists a toppling sequence leading to $c_{2}$. Note that being reachable is a transitive relation, i.e. $c_{1} \rightarrow c_{2}, c_{2} \rightarrow c_{3} \Rightarrow c_{1} \rightarrow c_{3}$.

Theorem 8. ([21],[8]) Given any configuration $c$, there exists a unique stable configuration $\sigma(c)$ such that $c \vdash \sigma(c)$, independent of the toppling sequence chosen.

Property 9. If $c \vdash \sigma(c)$, then $k c \vdash k \sigma(c)$

Property 9 will later be extensively used for proving gluing properties of potential functions. Associated with every toppling sequence is the count of the number of times each site has toppled, the vector of toppling potentials, also referred to as the score vector in [3]. These toppling potentials are very closely related to the electric potentials that develop at various nodes when power source-sink are appropriately applied, a connection which we will discuss in detail in the coming sections.

Definition 10. Assuming $c_{1} \vdash c_{2}$, the toppling potential function $z^{c_{1}, c_{2}}: V_{0} \rightarrow \mathbb{Z}^{+}$is defined as $z^{c_{1}, c_{2}}(v)$ : the number of times $v$ toppled in a toppling sequence from $c_{1}$ to $c_{2}$. We denote $z^{c, \sigma(c)}$ by $z^{c}$.

This function is well defined as the number of times a particular site topples is independent of the toppling sequence chosen, already noted in [3]. A simple proof follows from typical linear algebraic arguments and the fact that the principal minor of a connected graph's combinatorial Laplacian is of full rank.

A configuration is called recurrent if it is reachable from any configuration. As already mentioned, we say that a configuration $c_{i}$ is reachable from a configuration $c_{j}$ if by adding some particles to $c_{j}$ and subsequently relaxing it, we can obtain $c_{i}$. A configuration is transient if it is not recurrent. The set of recurrent configurations is therefore, closed under being reachable.

Definition 11. A configuration $c$ is recurrent iff $\forall c^{\prime}$ we have $c^{\prime} \rightarrow c$. 
Since recurrence persists under particle addition, we have the following property.

Property 12. If $c_{1} \leqslant c_{2}$, then recurrence of $c_{1}$ implies that of $c_{2}$.

Denote the configuration in which every node $v$ has degree $(v)$ particles by $c_{\max }$. Clearly, given any stable configuration $c$, one can reach $c_{\max }$ simply by adding the required number of particles at each site.

We analyze the process of adding one grain at a time to the sandpile and study its evolution. As in the standard theory of Markov chains, recurrence characterizes the long term (steady state) behavior of sandpiles. Our investigation is concerned with the maximum number of particles that can be added while staying transient. Following Babai and Toumpakari [4], we define the notion of the transience class as follows.

Definition 13. The transience class of $S$ denoted by $\operatorname{tcl}(S)$, is defined as the maximum number of particles that can be added to $S$ before reaching a recurrent configuration.

Remark: In [10], the authors talk about an alternate equivalent characterization of transience class which defines the transience class alternatively as the maximum number of particles that can be added before all the nodes have toppled at least once. Even though we will not be using this definition explicitly, the notion is inherent in the way we bound transience classes. We will be computing the maximum number of particles that can be added at any point before at least one particle reaches every node. For sandpile with bounded node degrees, using the Property (9), this translates to the alternate characterization of transience classes we just mentioned (albeit with a constant multiplicative loss factor, the degree).

\section{Basic Properties}

Consider any (possibly infinite) graph $G(V, E)$. Distances in this graph will henceforth correspond to the shortest path metric. For any vertex $v, B(v, r)$ denotes the ball of radius $r$ around $v$, i.e. the set of all vertices in $G$ which are at a (shortest path) distance of at most $r$ from $v$. For any set of vertices $U \subseteq V$, define two notions of boundary

- vertex-boundary, $\delta_{V} U$ is the set of vertices, in $U$, having neighbors in the set $V-U$.

- edge-boundary, $\delta_{E} U$ is the set of edges connecting vertices in $U$ to those in $V-U$.

For example if the set $U$ is the ball $B(v, r)$, the set $\delta_{V} B(v, r)$ is the set of vertices which are exactly at distance $r$ from $v$ and the set $\delta_{E} B(v, r)$ is the set of edges between the vertices in $\delta_{V} B(v, r)$ and those in $\delta_{V} B(v, r+1)$. Consider any connected set of vertices $V_{h}$ and the induced subgraph $G_{h}=\left(V_{h}, E_{h}\right)$. The sandpile corresponding to this subgraph is obtained by adding the edge set $\delta_{E} V_{h}$ to $E_{h}$ and identifying all the vertices in $V-V_{h}$ which have neighborhood in $V_{h}$ with the sink node $s$. We denote this sandpile by $S=\left(V_{h} \cup\{s\}, E_{h} \cup \delta_{E} V_{h}\right)$. 
An example of the grid sandpile: Consider the infinite grid graph with its canonical embedding in the plane. For every pair of integers $(i, j)$ there exists a vertex (with this pair as its label). Each vertex $(i, j)$ is adjacent to $(i+1, j),(i, j+1),(i-1, j)$ and $(i, j-1)$, which are the four lattice points flanking $(i, j)$. Let $V_{m, n}$ be the set of vertices with labels $\{(i, j): 0 \leqslant i \leqslant m, 0 \leqslant j \leqslant n\}$. Evidently this set is connected. The boundary vertex set is the set of vertices lying on the horizontal line segments $\{(i, 0): 0 \leqslant i \leqslant m\}$ and $\{(i, n): 0 \leqslant i \leqslant m\}$, and on the vertical ones $\{(0, j): 0 \leqslant j \leqslant n\}$ and $\{(m, j): 0 \leqslant j \leqslant n\}$. The edge-boundary set is the set of edges between vertices in the boundary set and the vertices not in the $(m+1) \times(n+1)$ block $\left(V_{h}\right)$. Figure 1 exhibits the graph, the aforementioned boundary sets and the resulting sandpile. In the left figure, the white nodes belong to the set $V-V_{m, n}$. The grey nodes form the vertex boundary of $V_{m, n}$, and along with the black nodes constitute the set $V_{m, n}$. The heavy edges constitute the edge boundary. The figure on the right is the sandpile $S_{m, n}$. The heavy edges are connections to the special sink node.

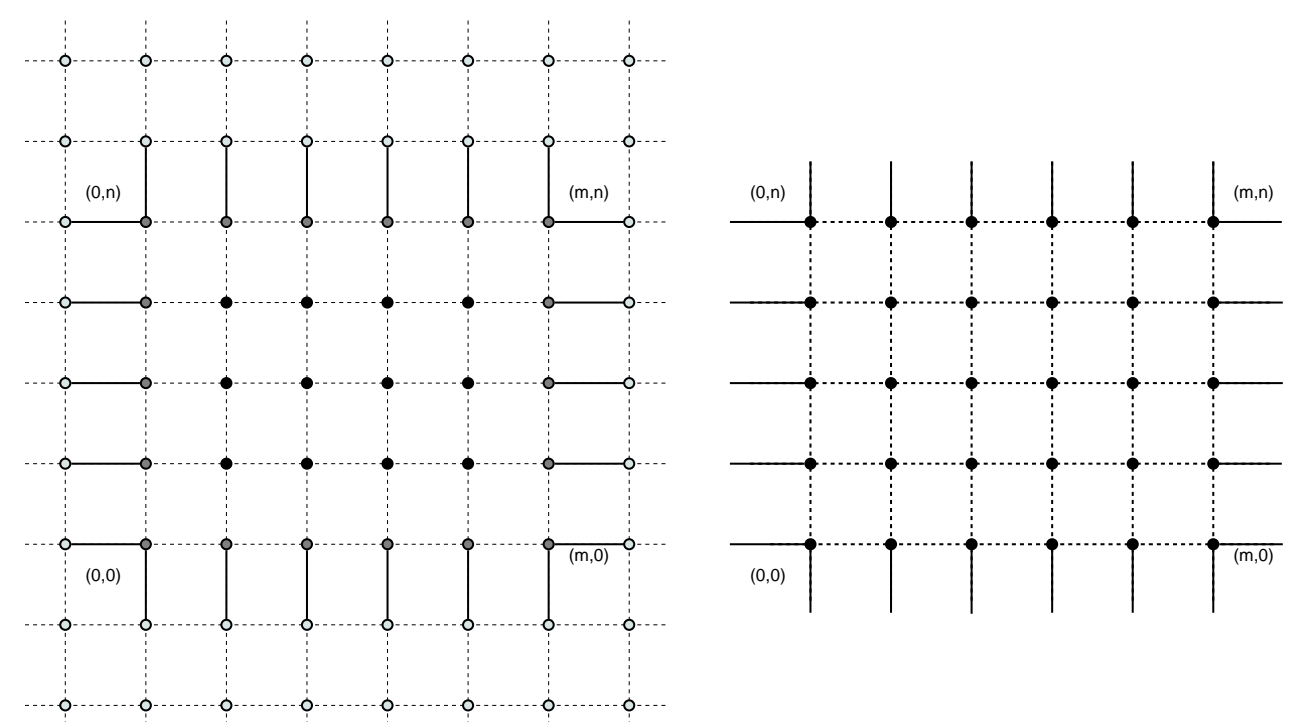

Figure 1: A grid graph, the subset set $V_{m, n}$ and the corresponding sandpile

We will denote both the sandpile and the underlying graph by the same symbol $S$. To keep notation and definitions clean, we fix the following convention. The ball $B(v, r)$ with respect to the shortest path metric is defined already. In the context of sandpile, however, when we say a ball $B(v, r)$ in some vertex subset $V_{h}$, or the induced sandpile $S$, we assume it is small enough to not contain the sink node. Also, whenever a sandpile is mentioned without explicit description of an ambient graph, we assume that a super-graph indeed exists and the sandpile has been obtained in the manner described above. Let $\mathcal{S} \equiv\left\{S_{i}\right\}$ be an indexed family of sandpile graphs. We will now define some basic properties of these (possibly infinite) families which we will need later in discussion on bounding the transience class. In the sequel, the volume of a ball $B(v, r)$ denotes the number of edges inside it and will be written as $\operatorname{Vol}(B(v, r))$. 
Definition 14. Polynomial Volume Growth Property $\left(V_{\alpha}\right)$ : A graph family $\mathcal{S}$ has polynomial volume growth property $\left(V_{\alpha}\right)$ with growth exponent $\alpha$ if there exist constants $\delta$ and $\Delta$ such that for any graph $S_{i} \in \mathcal{S}$ and every node $v \in V\left(S_{i}\right)$, if we consider the $B(v, r)$ around $v$, its volume satisfies the following bounds:

$$
\delta r^{\alpha} \leqslant \operatorname{Vol}(B(v, r)) \leqslant \Delta r^{\alpha} .
$$

This property ensures that there is a bound on the rate at which the measure grows. This becomes crucial if one wants to make any arguments along the lines of diffusions in Euclidean spaces. An easy consequence is the degree bound on the graph family $\mathcal{S}$. All vertices in every member graph have degrees bound between $\delta$ and $\Delta$.

Definition 15. Degree boundedness $(\Delta)$ : A graph family $\mathcal{S}$ satisfies $(\Delta)$ if for every graph $S_{i} \in \mathcal{S}$, the degree of every node is bounded from above by $\Delta$. Moreover, a sandpile satisfies $(\Delta)$ if every normal (non-sink) node has degree bounded by $\Delta$.

If adding $x$ particles at some node $u$ causes some node $v$ to receive a particle at any point in time, we say that $v$ is flooded. Similarly, we say that the set $V^{\prime} \subset V$ got flooded if all the nodes it consisted of, received at least one particle. There are two symmetry properties that will be important to us.

Definition 16. High Local-Conductance Property $\left(\mathrm{hLC}\left(C_{\sigma}\right)\right)$ : A sandpile family $\mathcal{S}$ satisfies hLC $\left(C_{\sigma}\right)$ if there exists a constant $C_{\sigma}$, independent of the index $i$ of the sandpile $S_{i} \in \mathcal{S}$, such that for any site $v \in V\left(S_{i}\right)$ and any ball $B(v, r)$ in $S_{i}$, placing $C_{\sigma} \cdot \operatorname{Vol}(B(v, r))$ particles at $v$ floods the ball $B(v, r)$.

This property limits the amount of dissipation through the boundary of the sandpile, as long as we restrict ourselves to flooding balls that lie completely inside the set of ordinary vertices. We will be needing the definition of harmonic functions for describing the next property. Given a connected graph $G$ and a function $\pi: V(G) \rightarrow \mathbb{R}$, we say that $\pi$ is harmonic over the vertex set $V_{h}$ if,

$$
\frac{1}{\operatorname{degree}(v)} \sum_{u \sim v} \pi(u)=\pi(v), \quad v \in V_{h}
$$

The vertices in $V-V_{h}$, adjacent to any vertex in $V_{h}$, are called the "poles" of $\pi$. The set $V_{h}$ is also called the interior of $\pi$ and the set of poles referred to as the boundary. Being harmonic over $V_{h}$ means that the value of $\pi$ at any vertex in $V_{h}$ is the average of its value in the immediate neighborhood. In case of multigraphs, we take the appropriate weighted means, where the weights are the number of common edges. For a slightly expanded discussion on some essential properties of harmonic functions, see appendix A. For a proper introduction to harmonic functions on graphs, we refer the reader to the beautiful paper by Benjamini and Lovasz [6]. See Telcs [46] for a thorough review.

Definition 17. Mean Value Property $\left(\operatorname{MV}\left(C_{h}\right)\right)$ : A sandpile family $\mathcal{S}$ satisfies $\operatorname{MV}\left(C_{h}\right)$ if there exists a constant $C_{h}$, independent of the index $i$ of the sandpile $S_{i} \in \mathcal{S}$, such that 
for any graph $S_{i}=\left(V_{h} \cup\{s\}, E_{h} \cup \delta_{E} V_{h}\right)$ if we consider any function $\pi: V\left(S_{i}\right) \rightarrow[0,1]$ defined over $V\left(S_{i}\right)$ which is harmonic over a ball $B(v, r) \subset V_{h}$, the following inequality holds,

$$
\sum_{u \in B(v, r)} \pi(u) \geqslant C_{h} \pi(v) \operatorname{Vol}(B(v, r))
$$

The continuous version of the mean value property stated above occupies central position in classical analysis. See [12] for further discussion on the importance of this assumption. We now define the local superposition property.

Definition 18. Local Superposition Property $\left(\operatorname{LS}\left(C_{l}\right)\right)$ : A sandpile family $\mathcal{S}$ satisfies $\mathrm{LS}\left(C_{l}\right)$ if there exists a constant $C_{l}$, independent of the index $i$ of the sandpile $S_{i} \in \mathcal{S}$, such that given a ball $B(v, R) \subset S_{i}$, if placing $H$ particles at $v$ topples a site $w$ in $B(v, R)$, then placing $h$ particles at every site in the smaller ball $B(v, r)$ also topples $w$, where,

$$
h=\frac{C_{l} H}{\operatorname{Vol}(B(v, r))} .
$$

Remark: This property essentially means that if placing $H$ particles at a site $v$ causes a toppling at site $w$, then the same effect can be produced at $w$ by adding a constant factor times $H$ particles distributed evenly among the sites in the ball $B(v, r)$. Such impulse distribution properties are easy to prove in the setting of classical complex analysis. The goal of proving a much more general superposition property, where the impulse is distributed not over a ball but a general set of nodes which are distance wise (in potential theoretic sense) well distributed with respect to the point of observation, seems unlikely to work out. There are simple counterexamples where such general distributivity does not work. However, it is also not known if the constraint of taking nodes inside a ball is essential.

Theorem 19. Given a sandpile family $\mathcal{S}$ which satisfies $M V\left(C_{h}\right)$ and $(\Delta)$, it also satisfies $L S\left(C_{l}\right)$. Moreover, the constants $C_{l}, C_{h}$ and $\Delta$ satisfy the following relation.

$$
C_{l}=\frac{\Delta+1}{C_{h}} .
$$

We defer the proof of this theorem to section 5 .

Definition 20. Overlapping Potentials Property $(\mathrm{OP}(f(\cdot)))$ : Given a sandpile family $\mathcal{S}$. Consider a member $S_{i}$ and let $h$ be the smallest number of particles which when placed at every site in a ball $B(v, r) \subseteq S_{i}$, makes every site in the ball $B(v, R) \subseteq S_{i}$ topple at least once. If $h$ is bounded by a function $f$ of $R / r$, independent of the sandpile index $i$, $\mathcal{S}$ satisfies the overlapping potentials property, $\mathrm{OP}(f(\cdot))$.

Lemma 21. Given a sandpile family $\mathcal{S}$ which satisfies $h L C\left(C_{\sigma}\right), M V\left(C_{h}\right)$ and $\left(V_{\alpha}\right)$, it also satisfies $\operatorname{OP}(f(\cdot))$. Moreover, the function $f$ has the following form.

$$
f(R, r)=\frac{C_{\sigma}}{C_{h}} \frac{\Delta(\Delta+1)}{\delta}\left(\frac{R}{r}\right)^{\alpha} .
$$


Proof. Given a sandpile family $\mathcal{S}$ which satisfies $\mathrm{hLC}\left(C_{\sigma}\right), \operatorname{MV}\left(C_{h}\right)$, and $V_{\alpha}$. Using theorem 19, it also satisfies $\operatorname{LS}\left(C_{l}\right)$. Consider a sandpile $S_{i} \in \mathcal{S}$. Consider any vertex $v \in V\left(S_{i}\right)$ with the balls $B(v, r)$ and $B(v, R)$ around it with $R>r$. Let $x$ be the minimum number of particles one needs to place at $v$ to topple every site in $B(v, R)$ at least once. Since $S$ satisfies $\mathrm{hLC}\left(C_{\sigma}\right)$, we have

$$
x \leqslant C_{\sigma} \Delta R^{\alpha} .
$$

The property $\left(V_{\alpha}\right)$ implies the following lower bound on the volume of the ball $B(v, r)$.

$$
\operatorname{Vol}(B(v, r)) \geqslant \delta r^{\alpha}
$$

Now, consider any site $p$ in $\delta B(v, R)$. Using $\operatorname{LS}\left(C_{l}\right)$, if $x$ particles placed at $v$ induce a toppling at $p$, then $C_{l} x / \operatorname{Vol}(B(v, r))$ particles at each of the sites in $B(v, r)$ necessarily induce a toppling at $p$. So, the minimum number of particles needed to be placed at each node of $B(v, r)$, to topple every site in $B(v, R)$ at least once, satisfy the following bound,

$$
h \leqslant C_{l} C_{\sigma} \frac{\Delta}{\delta}\left(\frac{R}{r}\right)^{\alpha} .
$$

Again, using theorem 19, $C_{l}=(\Delta+1) / C_{h}$. This gives us the final form of the polynomial.

$$
h \leqslant \frac{C_{\sigma}}{C_{h}} \frac{\Delta(\Delta+1)}{\delta}\left(\frac{R}{r}\right)^{\alpha} .
$$

Therefore, $h$ is indeed bounded by a polynomial in the ratio $R / r$.

Notation: With each site $v$ in a sandpile $S$, we associate the number $\eta(v)=\operatorname{dist}\left(v, \delta_{V}(S)\right)$ i.e. the distance between site $v$ and the vertex boundary of $S$. It is also the radius of the largest ball centered at $v$ which is inside $S$.

Lemma 22. (Epicenter Propagation : Single Step) Given a sandpile family $\mathcal{S}$ which satisfies $O P(f(\cdot))$. Let $v$ be a node in $S_{i} \in \mathcal{S}$, with $B(v, \eta(v))$ as the largest ball around it. Let $u$ be any site in $\delta_{V} B(v,\lfloor\eta(v) / 2)\rfloor$ (i.e. at distance $\lfloor\eta(v) / 2\rfloor$ from $v$ ). Then, there exists a constant $K$, independent of the sandpile index $i$, such that if a configuration $c$ floods the ball $B(v, \eta(v))$, then configuration K.c floods $B(u, \eta(u))$.

Furthermore, if the sandpile family $\mathcal{S}$ satisfies $h L C\left(C_{\sigma}\right), M V\left(C_{h}\right)$ and $\left(V_{\alpha}\right)$, then the constant $K$ satisfies the following bound.

$$
K \leqslant \frac{C_{\sigma}}{C_{h}} \frac{\Delta(\Delta+1)}{\delta} 3^{\alpha} .
$$

Proof. In the following proof, we will be assuming that all the quantities are integers. The argument without this simplification needs no new ideas and can be essentially reconstructed from the given proof.

Let the configuration $c$ be such that it floods the ball $B(v, \eta(v))$. Since $B(u, \eta(v) / 2) \subset$ $B(v, \eta(v)), c$ also floods $B(u, \eta(v) / 2)$. Let $K$ be the number of particles needed at every 


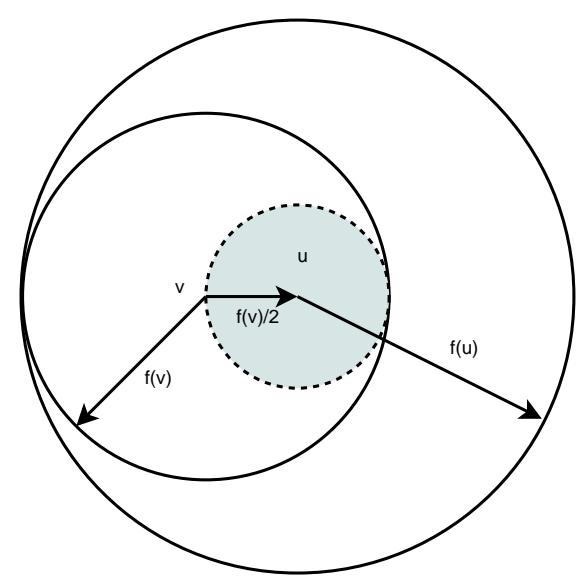

(a)

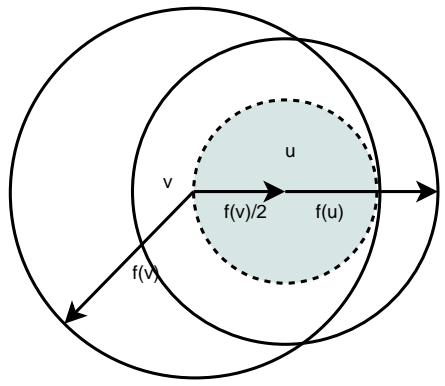

(b)

Figure 2: Epicenter Propagation : Single step (a) Expansion (b) Contraction

site in the ball $B(u, \eta(v) / 2)$, to flood the normal ball $B(u, \eta(u))$. Using the overlapping potentials property $(\mathrm{OP}), K$ is bounded by a polynomial in $2 \eta(u) / \eta(v)$. The equation (4) illustrates this polynomial. Putting in the appropriate value of radii, we get the following polynomial.

$$
K \leqslant \frac{C_{\sigma}}{C_{h}} \frac{\Delta(\Delta+1)}{\delta}\left(\frac{\eta(u)}{\eta(v) / 2}\right)^{\alpha} .
$$

In the sandpile $S_{i} \in \mathcal{S}$, consider any shortest path connecting $v$ to $u$, where $u \in$ $\delta_{V} B(v, \eta(v) / 2)$. The value of $\eta$ can vary by at most one with every step. Therefore, $\eta(v) / 2 \leqslant \eta(u) \leqslant 3 \eta(v) / 2$. In particular, the ratio of radii is bounded.

$$
\frac{\eta(u)}{\eta(v) / 2} \leqslant 3
$$

Substituting the above bound on radius ratio, we obtain the expression in equation (5). Using Property 9, K.c places $K$ particles on each site of $B(u, \eta(v) / 2)$, which in turn necessarily floods $B(u, \eta(u))$. The expression in equation (5) is clearly independent of the sandpile index $i$. This completes the proof.

Note that there are two distinct case here, $\eta(u) \leqslant \eta(v)$ and $\eta(u) \geqslant \eta(v)$. Even though one argument suffices to obtain bounds for both the cases, they are qualitatively different. See Figure (2). The case in which the distance from the sink node is increasing along the path is termed the expansion step and the other case is called the contraction step. There is also a third variant, the drift step. The need for such distinction will become clear when we prove the general multi-step version of this lemma in the next section.

Remark: The above lemma allows one to shift the focal point of diffusion by some distance at the cost of an additional multiplicative constant. This generalizes the traveling 
diamond lemma of Babai and Gorodezky [3]. This lemma forms the backbone of the main result. While proving the polynomial bounds on the transience class, we will be using this lemma to keep shifting the epicenter from some starting node to a target node. We will next bound the total number of applications of this lemma by $O(\log n)$. This will imply an $O(\operatorname{poly}(n))$ bound on the number of particles required to shift the epicenter between any pair of sites.

\section{Bounding the transience class}

In the proof of the Lemma (22), we use the fact that along any shortest path joining a vertex $v$ to a vertex in $B(v, r)$ (lying inside the sandpile), the distance from sink cannot increase or decrease too fast. For our purpose, this precondition of $u$ belonging to $B(v, r)$ is too restrictive. We will impose a more general constraint on the graph structure using the non-empty interior (NI) property for paths in graphs. Before that, we will need some basic definitions. If $P$ is path from $u$ to $v$, and $w \in P$ is some vertex on that path, then $\operatorname{dist}_{P}(w, u)$ is the distance of $w$ from $u$ along the path $P$. So, if $P=\left\{u_{0} \cdot u_{1}, \ldots, u_{k}\right\}$ then $\operatorname{dist}_{P}\left(u_{0}, u_{i}\right)=i$.

Definition 23. The function $f: V \rightarrow \mathbb{R}$ varies linearly over path $P$ (from $v_{1}$ to $v_{2}$ ) if for any site $v$ at a distance $\operatorname{dist}_{P}\left(v_{1}, v\right)$ along $P$ from $v_{1}$, we have the following two-sided bounds on $f(v)$.

$$
a_{l}+f\left(v_{1}\right)+b \operatorname{dist}_{P}\left(v_{1}, v\right) \leqslant f(v) \leqslant a_{u}+f\left(v_{1}\right)+b \operatorname{dist}_{P}\left(v_{1}, v\right)
$$

Here, $a_{u}, a_{l}$ and $b$ are constants with absolute values bounded by 1 .

Note: Consider the $\eta$ function defined earlier ${ }^{1}$. For this function, the value of $b$ lies in the closed interval $[-1,1]$ as the distance from sink cannot increase (or decrease) by more then one, when one step is taken along the path.

Consider a sandpile family $\mathcal{S}$ and a member graph $S_{i}$. Call a path $P$ from $v_{1}$ to $v_{2}$ $(1, l)$-central if $\eta\left(v_{i}\right)$ values vary linearly over $P$, or if its length is bounded by $l \log \left(\left|S_{i}\right|\right)$. We call it $(k, l)$-central if it can be split into at most $k$ central paths, where $k$ and $l$ are constants independent of graph index (and size).

Definition 24. Non-empty Interior Property (NI) : A sandpile family $\mathcal{S}$ has non-empty interior property, $\mathrm{NI}(k, l)$, if for every member sandpile graph $S_{i} \in \mathcal{S}$ and if for every pair of vertices $v, w \in V\left(S_{i}\right)$, there exists a path between them which is $(k, l)$-central. Here, $k$ and $l$ are constants independent of the sandpile index $i$.

Definition 25. Polynomial Transience Class Property (pTcl) : We say that the transience class of a sandpile family $\mathcal{S}$ is polynomially bounded if, for any member sandpile $S_{i} \in \mathcal{S}$, adding at most polynomial (in sandpile volume $\left|S_{i}\right|$ ) particles at any site induces a toppling at every other site.

\footnotetext{
${ }^{1} \eta(u)$ is the distance of node $u$ from the sink node minus one, i.e., it equals the radius of the largest ball centered at $u$ which doesn't intersect with the sink node
} 
The following lemma proves the polynomial bound on transience class of certain sandpile families. It is a generalization of the expansion and contraction phases while flooding a grid, as mentioned in Babai and Gorodezky [3].

Theorem 26. (Epicenter Propagation Theorem) Given a sandpile family $\mathcal{S}$ which satisfies $h L C\left(C_{\sigma}\right), O P(f(\cdot))$ and $N I((k, l))$. Then $\mathcal{S}$ satisfies $p T c l$.

Moreover, if $\mathcal{S}$ also satisfies $M V\left(C_{h}\right)$ and $V_{\alpha}$, then for any member sandpile $S_{i} \in \mathcal{S}$, the transience class satisfies the following bounds

$$
\operatorname{tcl}\left(S_{i}\right) \leqslant C_{\sigma} \Delta n^{k+(2 l+1) k \log _{\hat{g}}\left(\frac{C_{\sigma}}{C_{h}} \frac{\Delta(\Delta+1)}{\delta} 3^{\alpha}\right)}
$$

Here, $n=\left|S_{i}\right|$.

Proof. First consider the case $k=1$. The general case will follow immediately by taking the $k^{\text {th }}$ power of particles required in the special case. Consider the sandpile $S_{i} \in \mathcal{S}$, and a pair of vertices $p$ and $q$ with a $(1, l)$-central path $P$ from $p$ to $q$. Denote the vertices on the path $P$ as $v_{0}=p, v_{1}, \ldots, v_{n}=q$. The value of $f$ changes linearly from $\eta(p)=a$ to $\eta(q) \approx a+b n$. There are three essentially different cases which can arise, $b>0$ and $b<0$, when $\eta$ varies linearly, and path length is logarithmic in graph size, when $\eta$ varies sub-linearly. We consider them individually.

1. Case $(b>0)$ Expansion Phase : Refer to Figure (3). Initialize by adding some constant number of particles so that the normal ball $B\left(v_{3}, 3\right)$ is flooded. Let this constant be $K_{0}$. We will now repeat the following process iteratively till $q$ receives a particle.

Assume that we are currently at vertex $v_{i}$ and the normal ball $B\left(v_{i}, \eta\left(v_{i}\right)\right)$ is flooded. Consider the vertex $v_{j}$ with $j=i+\eta\left(v_{i}\right) / 2$ and the normal balls $B_{s}=B\left(v_{j}, \eta\left(v_{i}\right) / 2\right)$ and $B_{t}=B\left(v_{j}, \eta\left(v_{j}\right)\right)$. $B_{s}$ was flooded in the last iteration. We use this to flood the concentric ball $B_{t}$. Using Lemma (22), if configuration $c$ flooded $B\left(v_{i}, \eta\left(v_{i}\right)\right)$, then $K . c$ floods $B\left(v_{j}, \eta\left(v_{j}\right)\right)$ for constant $K$. In every iteration, the radius of the target ball $B_{t}$ increases by at least a factor of $\eta\left(v_{j}\right) / \eta\left(v_{i}\right)$.

$$
\frac{\eta\left(v_{j}\right)}{\eta\left(v_{i}\right)}=\frac{a+b j}{a+b i}
$$

Since, $j=i+\eta\left(v_{i}\right) / 2$ we get,

$$
\frac{\eta\left(v_{j}\right)}{\eta\left(v_{i}\right)}=\frac{a+b i+\eta\left(v_{i}\right) / 2}{a+b i} .
$$

Separating out into two summands, we get,

$$
\begin{aligned}
\frac{\eta\left(v_{j}\right)}{\eta\left(v_{i}\right)} & =\frac{a+b i}{a+b i}+\frac{\eta\left(v_{i}\right) / 2}{\eta\left(v_{i}\right)} \\
& =1+b / 2
\end{aligned}
$$


Let us define $g=1+b / 2$. The path length is bounded from above by $n=\left|V\left(S_{i}\right)\right|$. Therefore, the total number iterations is at $\operatorname{most} \log _{g}(n)$. Each step contributes a bounded multiplicative factor to the total count of particles needed. This implies that the total number of particles needed, say $N$, is bounded by the following expression.

$$
N \leqslant K_{0} K^{\log _{g}(n)}=K_{0} n^{\log _{g}(K)}
$$

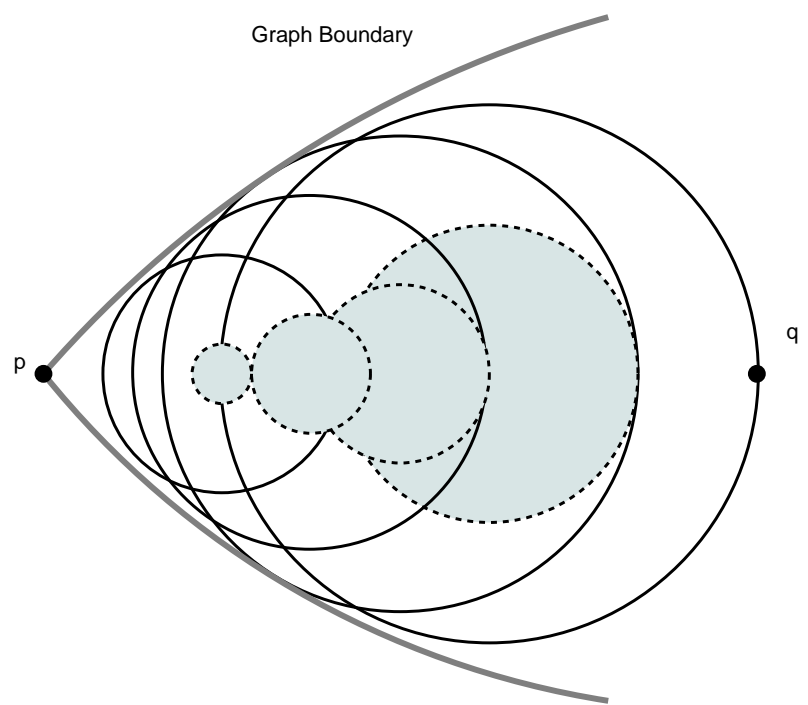

Figure 3: Epicenter Propagation : Expansion Phase - the radii of balls are expanding and the potential focus is moving towards the central region in the graph

2. Case $(b<0)$ Contraction Phase: This case is analogous to the Expansion phase. See Figure (4). We initialize by flooding the ball $B(p, \eta(p))$. Using the property $\mathrm{hLC}\left(C_{\sigma}\right)$, the number of particles required, say $K_{0}^{\prime}$, is bounded by $C_{\sigma} \cdot \operatorname{Vol}(B(p, \eta(p)))$. But the volume of this ball is trivially bounded by the volume of the sandpile $S_{i}$, which in turn is trivially bounded by product of the maximum degree, $\Delta$, times the total number of vertices, $n$.

$$
K_{0}^{\prime} \leqslant C_{\sigma} \Delta n
$$

We will now repeat the following process iteratively. Assume that we are currently at vertex $v_{i}$ and the normal ball $B\left(v_{i}, \eta\left(v_{i}\right)\right)$ is flooded. Consider the vertex $v_{j}$ with $j=i+\eta\left(v_{i}\right) / 2$ and the normal balls $B_{s}=B\left(v_{j}, \eta\left(v_{i}\right) / 2\right)$ and $B_{t}=B\left(v_{j}, \eta\left(v_{j}\right)\right)$. In the last iteration, $B_{s}$ was flooded along with the ambient $B\left(v_{i}, \eta\left(v_{i}\right)\right)$. Using $B_{s}$, we will to flood the concentric ball $B_{t}$. Again, using Lemma (22), if configuration $c$ flooded $B\left(v_{i}, \eta\left(v_{i}\right)\right)$, then $K . c$ floods $B\left(v_{j}, \eta\left(v_{j}\right)\right)$ for some constant $K$. In every iteration, the radius of the target ball $B_{t}$ decreases by a factor of $\eta\left(v_{j}\right) / \eta\left(v_{i}\right)$.

Following through the same computation as in the case of expansion phase, we obtain that the contraction ratio is $1+b / 2$. Note that, since $b<0$, this ratio is less 
than 1. There is a constant factor contraction in the radius of the balls flooded in each iteration. Let us denote the reciprocal of this ratio by $g^{\prime}=(1+b / 2)^{-1}$. The implication of this remains the same though, that the total number of iterations are logarithmic in $n$. To be precise, the number of iterations is at most $\log _{g^{\prime}}(n)$. Since each step contributes a multiplicative factor to the total particle requirement, the total number of particles needed, say $N$, is bounded by the following expression.

$$
N \leqslant K_{0}^{\prime} K^{\log _{g^{\prime}}(n)}=K_{0}^{\prime} n^{\log _{g^{\prime}}(K)}
$$

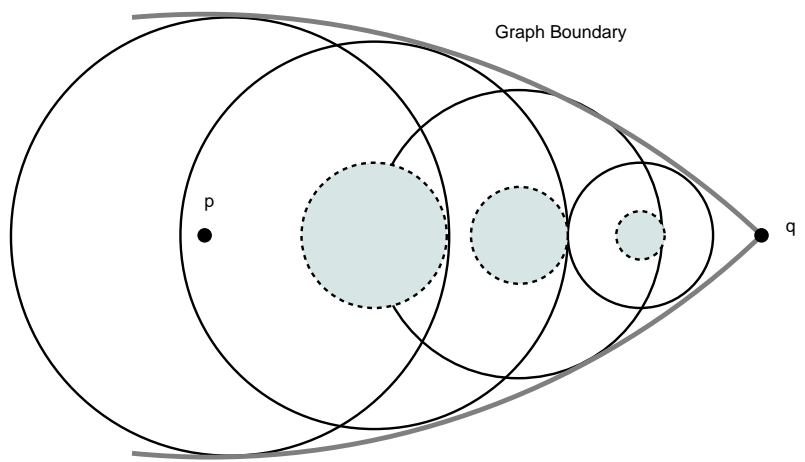

Figure 4: Epicenter Propagation : Contraction Phase - the radii of balls are decreasing and the potential focus is moving towards the boundary region

3. Case $(|P|=O(\log (n)))$ Lateral Drift Phase: Let the path $P$ be such that $\eta$ varies sub-linearly over it. Also, let the minimum value of $\eta$ over $P$ be $\eta_{\min }$. Consider the ball $B\left(p, \eta_{\text {min }}\right)$. We flood this using at most $K_{0}^{\prime} \leqslant C_{\sigma} \Delta n$ particles (follows from the (hLC $\left.\left(C_{\sigma}\right)\right)$ property, just like the previous case).

If configuration $c$ floods $B\left(p, \eta_{\min }\right)$, then $K c$ floods $B\left(v_{\eta_{\min } / 2}, \eta_{\min }\right)$, using Lemma (22). At a multiplicative cost of $K$, we cover a distance of $\eta_{\min } / 2$ on the path $P$. Let $l$ be a constant, independent of the sandpile index, such that $|P| \leqslant l \cdot \log n$. We take at most $2 l \log n / \eta_{\min }$ steps in this fashion. See Figure (5). The total number of particle required to finally flood the site $q$, say $N$, is bounded by the following expression:

$$
N \leqslant K_{0}^{\prime} K^{\frac{2 l}{\eta_{m i n}} \log (n)}=K_{0}^{\prime} n^{\frac{2 l}{\eta_{m i n}} \log (K)}
$$

Noting that $\eta_{\min } \geqslant 1, K_{0} \leqslant K_{0}^{\prime}=C_{\sigma} \Delta n$, and denoting $\hat{g}=\min \left\{g, g^{\prime}\right\}$, we have the following common bound on $N$ which respects the bounds in equations (8), (9), and (7).

$$
N \leqslant C_{\sigma} \Delta n^{1+(2 l+1) \log _{\hat{g}}(K)}
$$

After considering these cases of central paths, the argument of $k$-central paths follows directly. We split the path in the central components. Each of these components contributes a polynomial factor, mentioned above, in the total particles count. Since $k$ is a 


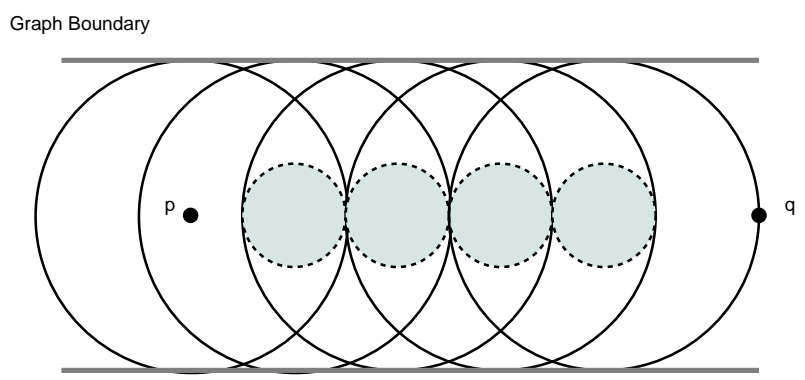

Figure 5: Epicenter Propagation : Lateral Drift Phase - the radii of balls under consideration stay same but the potential focus drifts inside the graph

constant for the family of sandpile under consideration, we obtain that the total particle count is the following polynomial.

$$
N \leqslant C_{\sigma} \Delta n^{k+(2 l+1) k \log _{\hat{g}}(K)}
$$

Now assuming that the conditions $M V\left(C_{h}\right)$, and $V_{\alpha}$ are also satisfied, and using bounds on the value of $K$ as mentioned in the Lemma (22), the bounds on $N$ can be written as,

$$
N \leqslant C_{\sigma} \Delta n^{k+(2 l+1) k \log _{\hat{g}}\left(\frac{C_{\sigma}}{C_{h}} \frac{\Delta(\Delta+1)}{\delta} 3^{\alpha}\right)}
$$

This completes the derivation of the theorem.

The theorem demonstrates polynomial bounds on the transience class of certain sandpile families. Furthermore, it shows that the bounds are of the form

$$
N \leqslant k n^{p(\alpha)}
$$

where $k$ is a constant and $p(\alpha)$ is a linear function of $\alpha$, for the particular family. This form of bound can be contrasted with the Harnack's inequality, where the bounds on growth rates of harmonic functions look similar, with the dimension appearing in the exponent. Note that both the volume growth parameter $\alpha$ and the dimension of a Euclidean space, determine the measure contained in any region. Hence, the similarity confirms the intuition behind thinking of $\alpha$ as the dimension for certain kinds of graphs.

\section{$5 \quad$ Impulse Superposition in sandpile : Proof of theorem 19}

We will now show that the local superposition property $\operatorname{LS}\left(C_{l}\right)$ follows from mean value property $\operatorname{MV}\left(C_{h}\right)$ and degree boundedness $(\Delta)$ of the graph. Let $H$ be the maximum number of particles which can be added at site $v$ without causing a toppling at site $w$. The following theorem from [10] bounds the value of $H$ for degree-bounded sandpile.

Theorem 27. ([10]) For any sandpile with bounded vertex degrees, the minimum number of particles that need to be added at any vertex $v$ to observe a toppling at any vertex $w$, 
$H$, satisfies the following two-sided bounds,

$$
\frac{1}{(\Delta+1) \pi_{w}(v)} \sum_{u} \pi_{w}(u) \leqslant H \leqslant \frac{(\Delta-1)}{\pi_{w}(v)} \sum_{u} \pi_{w}(u) .
$$

Similarly, let $h$ be maximum number of particles which when placed at each site of the ball $B(v, r)$ do not topple $w$. Let $z(u)$ denote the number of times the node $u$ topples in the process. Once the sandpile is stable, we observe that the number of particles on any particular node $u$ is between 0 and $\operatorname{degree}(u)-1$. This number is equal to the difference between total inflow and outflow. The inflow is due to neighbors' toppling and direct particle additions at $u$, and the outflow is due the toppling of node $u$ itself. Writing out the equations in integer variables for each of these nodes and taking the rational relaxation (treating the integer variables as rationals) gives us the following LP, which bounds the value of $h$ from above.

$$
\begin{array}{r}
\max h \\
\forall u \in B(v, r): 0 \leqslant \sum_{u^{\prime} \sim u} z\left(u^{\prime}\right)-\operatorname{degree}(u) z(u)+h \leqslant \operatorname{degree}(u)-1 \\
\forall u \notin B(v, r): 0 \leqslant \sum_{u^{\prime} \sim u} z\left(u^{\prime}\right)-\operatorname{degree}(u) z(u) \leqslant \operatorname{degree}(u)-1 \\
z(w) \leqslant 0, z \geqslant 0, h \geqslant 0
\end{array}
$$

There are two sets of flow conservation equations here, one for the nodes inside $B(v, r)$ where particles have been added directly, and the second for the remaining nodes in $S-B(v, r)$. From the weak duality for LPs, it follows that to obtain an upper bound of $\alpha$ on the optimum value of the above system, it suffices to find a feasible solution of the dual LP of value $\alpha$. The following minimization program is the dual of the above.

$$
\begin{gathered}
\min \sum_{u}(\operatorname{degree}(u)-1) Y(u) \\
\sum_{u^{\prime} \sim w} Y\left(u^{\prime}\right)+Y^{\prime}-\operatorname{degree}(w) Y(w) \geqslant 0 \\
\forall u \neq w: \sum_{u^{\prime} \sim u} Y\left(u^{\prime}\right)-\operatorname{degree}(u) Y(u) \geqslant 0 \\
\sum_{u \in B(v, r)} Y(u) \geqslant 1 \\
Y \geqslant 0, Y^{\prime} \geqslant 0
\end{gathered}
$$

Now, consider the following set of equations:

$$
\begin{aligned}
\sum_{u^{\prime} \sim w} Y\left(u^{\prime}\right)+Y^{\prime}-\operatorname{degree}(w) Y(w) & =0 \\
\forall u \neq w: \sum_{u^{\prime} \sim u} Y\left(u^{\prime}\right)-\operatorname{degree}(u) Y(u) & =0 \\
\sum_{u \in B(v, r)} Y(u) & =1
\end{aligned}
$$


A non-negative set of values satisfying the above set is feasible for the dual LP. We find these by considering the resistive circuit $\widehat{S}$, obtained by replacing each edge in $S$ by a unit resistance. We assign ground potential to the sink, and inject current at node $w$ such that it gets unit potential. The potential that develops on any node $u$ is ${ }_{s} \pi_{w}(u)$. Evidently, all these potential values belong to the unit interval $[0,1]$. The sum of potential values at nodes in $B(v, r), \sum_{u \in B(v, r)} \pi_{w}(u)$, can be used to scale the input current at $w$ thereby scaling all the potentials as well, such that the sum of potentials over the set of nodes $u \in B(v, r)$ becomes unit. It follows that the values $Y(u)=\pi_{w}(u) / \sum_{u \in B(v, r)} \pi_{w}(u)$ and $Y^{\prime}$ equaling the value of the current injected form a feasible solution of the dual LP. This gives the following bound on $h$ in terms of the objective value at this point.

$$
h \leqslant \frac{1}{\sum_{u \in B(v, r)} \pi_{w}(u)} \sum_{u}(\operatorname{degree}(u)-1) \pi_{w}(u) .
$$

We are given that the sandpile graph satisfies the mean value $\operatorname{MV}\left(C_{h}\right)$ property (definition (17)). We restate the mean value inequality below.

$$
\sum_{u \in B(v, r)} \pi_{w}(u) \geqslant C_{h} \pi_{w}(v) \operatorname{Vol}(B(v, r))
$$

for some constant $C_{h}$ independent of the sandpile index. Using equations 11 and 12, we get the following bounds on $h$ (up to constant factors):

$$
h \leqslant \frac{1}{C_{h} \operatorname{Vol}(B(v, r))} \frac{1}{\pi_{w}(v)} \sum_{u}(d(u)-1) \pi_{w}(u) .
$$

Now, using the lower bounds in equation 10, which tightly approximates the value of $H$, and equation 13, which bounds the value of $h$, we can bound $h$ in terms of $H$. The following relation is obtained:

$$
h \leqslant \frac{\Delta+1}{C_{h}} \frac{H}{\operatorname{Vol}(B(v, r))}
$$

The equation demonstrates the local superposition property $\operatorname{LS}\left(C_{l}\right)$. In particular, it shows that the constant $C_{l}$ of $\operatorname{LS}\left(C_{l}\right)$ is related to the constants $C_{h}$, of $\mathrm{MV}\left(C_{h}\right)$, and $\Delta$, of $(\Delta)$, in the following form:

$$
C_{l}=\frac{\Delta+1}{C_{h}}
$$

This completes the proof of theorem 19.

\section{The special case of grid sandpiles}

Figure (1) shows an example of an $m \times n$ grid based sandpile. We consider the family of sandpiles consisting of the symmetric case $(m=n)$. 


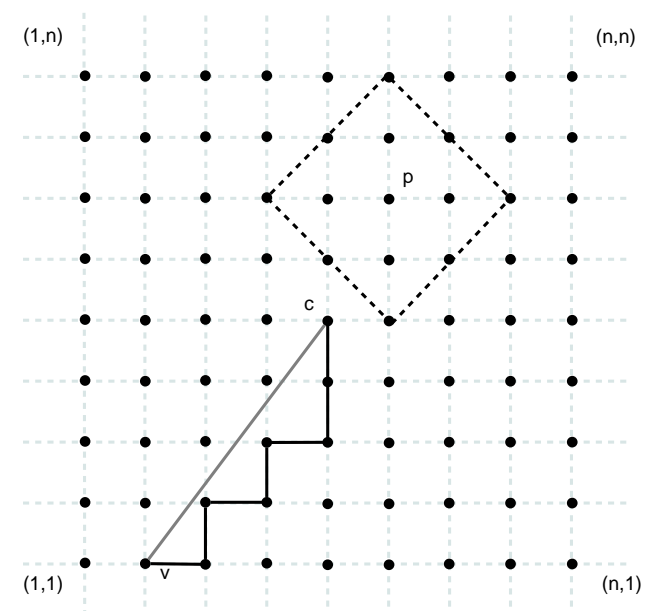

Figure 6: An $n \times n$ grid sandpile : $\operatorname{GRID}_{n}$

Consider an $n \times n$ grid graph. Attach an extra sink node $s$ to all the nodes lying on the boundary of this grid such that there is a double edge with every corner node and single edge for others. We denote the sandpile and graph by GRID ${ }_{n}$. Figure (6) shows an example.

As in the example of grid sandpile, we assume that our sandpile is coming from the induced subgraph over a suitable set of vertices in the infinite lattice. The reason behind this choice is that we will be talking about the family of all grid sandpiles. While a particular finite grid can be a subgraph of many infinite graphs, the infinite grid is the canonical choice of a common ancestor of all finite grid graphs. We will show in this section that the infinite grid, $\mathbb{Z}^{2}$, along with the finite subgraph GRID $_{n}$ satisfy all the properties required to imply polynomial bounds on the transience class of grids.

Degree bound $(\boldsymbol{\Delta})$ : The infinite grid $\mathbb{Z}^{2}$ is regular with degree 4 . Every induced subgraph obeys this degree bound.

Polynomial volume growth property $\left(\boldsymbol{V}_{\boldsymbol{\alpha}}\right)$ : For any vertex $p$ and radius $r$, volume of the ball $B(p, r)$ grows as a quadratic in $r$. That is to say $\mathbb{Z}^{2}$ satisfies $V_{\alpha}$ with $\alpha=2$. This simple fact can be proved using elementary counting arguments. Figure (6) shows a typical ball around vertex $p$.

Non-empty interior property $(\mathrm{NI}(2,0))$ : Given any finite grid, $\mathrm{GRID}_{n}$, it suffices to show that there exists a path from center to every other node on which the distance to sink varies linearly. For simplicity, assume that $n$ is odd and take any point $v$ on the boundary. Denote the center node by $c$. Consider the canonical embedding of this grid in the plane. Join $c$ to $v$ by a line segment. Take the projection of this line segment towards $x$-axis and construct the path using the set of highest lattice points lying below the segment. This path has the requisite property and the growth parameter is just the slope of the line joining $c$ and $v$. Figure (6) shows one such path. For an arbitrary pair of points $u, v$, the path is a juxtaposition of the paths from $u$ to $c$, and from $c$ to $v$. So at most two (1,0)-central components suffice. The argument for the more general case of $v$ lying inside the grid is identical. The case of even $n$ can be handled using essentially the 
same ideas.

Notation: We have been denoting the ball of radius $r$ (shortest path metric) around a node $v$ by $B(v, r)$. We will be extending this notation such that $B(v, r, R)$ will be taken to mean the annular region $B(v, R)-B(v, r)$.

Mean Value property (MV): For a proof of the mean value property (MV), we will be needing Lemma 6 from [28]. We reproduce it, with a slightly changed notation, below for reference.

Lemma 28. (Exact Mean Value property on an approximate ball, [28]) For each real number $r>0$, there is a function $w_{r}: \mathbb{Z}^{d} \rightarrow[0,1]$ such that,

- $w_{r}(x)=1$ for all $x \in B(v, r-c)$, for a constant $c$ depending only on $d$.

- $w_{r}(x)=0$ for all $x \notin B(v, r)$.

- For any function $\pi$ that is discrete harmonic on $B(v, r)$,

$$
\sum_{x \in \mathbb{Z}^{d}} w_{r}(x)(\pi(x)-\pi(v))=0 .
$$

This lemma allows us to get up to a constant distance of the boundary of the ball under consideration. We present a rough outline of the derivation of (MV) using this lemma. The reader is referred to appendix B for an elementary discussion on various volume inequalities in lattice graphs. Equations derived there will be used in the sequel.

Proof. (Outline) We will be denoting $B(v, r)$ by the shortened form $\mathbf{B}_{r}$, and $B(v, r, R)$ by $\mathbf{B}_{r, R}$. Using lemma (28) we have the following,

$$
\sum_{x \in \mathbf{B}_{r}} w(x) \pi(x)=\sum_{x \in \mathbf{B}_{r}} w(x) \pi(v)
$$

Decomposing the summation on both sides into a smaller ball and annular region, we obtain the following.

$$
\sum_{x \in \mathbf{B}_{r-c}} w(x) \pi(x)+\sum_{x \in \mathbf{B}_{r-c, r}} w(x) \pi(x)=\sum_{x \in \mathbf{B}_{r-c}} w(x) \pi(v)+\sum_{x \in \mathbf{B}_{r-c, r}} w(x) \pi(v)
$$

Using the property that $\forall x \in \mathbf{B}_{r-c}, w(x)=1$, and denoting the number of nodes in $\mathbf{B}_{r-c}$ by $\#_{V}\left(\mathbf{B}_{r-c}\right)$, we obtain the following.

$$
\sum_{x \in \mathbf{B}_{r-c}} \pi(x)+\sum_{x \in \mathbf{B}_{r-c, r}} w(x) \pi(x)=\pi(v)\left(\#_{V}\left(\mathbf{B}_{r-c}\right)+\sum_{x \in \mathbf{B}_{r-c, r}} w(x)\right)
$$

In equation (15), using the fact that $w(x) \leqslant 1$ for the second term on left hand side, and dropping the second term on the right hand side, we obtain

$$
\sum_{x \in \mathbf{B}_{r}} \pi(x) \geqslant \#_{V}\left(\mathbf{B}_{r-c}\right) \pi(v)
$$


Using equation (23), we obtain the following:

$$
\sum_{x \in \mathbf{B}_{r}} \pi(x) \geqslant \frac{1}{2^{k}} \#_{V}\left(\mathbf{B}_{r}\right) \pi(v) \quad \forall r>2 c
$$

Noting that $\operatorname{Vol}\left(\mathbf{B}_{r}\right)=4 \#_{V}\left(\mathbf{B}_{r}\right)$, since every node has degree 4 , we obtain the following form of the mean value inequality:

$$
\sum_{x \in \mathbf{B}_{r}} \pi(x) \geqslant \frac{1}{2^{k+2}} \operatorname{Vol}\left(\mathbf{B}_{r}\right) \pi(v) \quad \forall r>2 c
$$

Note: This property allows us to use balls with radii greater then $2 c$ (where $c$ is a function of lattice dimension alone). However this creates no essential problem. Using elementary combinatorial arguments, it is easy to show that if adding $N$ particles at some node $v$ causes the sand particles to reach within a distance $d$ from the sink node, then adding $k N$ particles at $v$ makes a particle reach the sink node, where $k$ is a function of $d$ and the dimension of lattice.

High local-conductance (hLC): Babai and Gorodezky [3] prove both (hLC) and (LS) property of grids, using elementary combinatorial arguments. We reproduce the discussion below without any essential changes. Note that the mean-value property is used in the Theorem 19, where it along with the property $\Delta$, implies the Local Superposition (LS) property. This is used in the proof of the Lemma 21 to prove the Overlapping Potentials (OP) property. Therefore showing (LS) directly obviates the need to prove (MV) altogether.

Definition 29. $D_{4}$ symmetry: A function $f: \mathbb{Z}^{2} \rightarrow \mathbb{N}$ is said to have $D_{4}$ symmetry with respect to a vertex $v$ if it is symmetric with respect to all the four axes of symmetry passing through $v$.

In case the function is defined on a finite $n \times n$ grid, the above definition is applied by assigning zero to all the points on which the function is not defined. This creates a function with a finite support. In this case, the point $v$ is bound to be the geometric center of this finite support.

Definition 30. Axis Monotonicity: A function $f: \mathbb{Z}^{2} \rightarrow \mathbb{N}$ is called axis monotone about some vertex $v$ if $f(p) \leqslant f(q)$ for any pair of lattice points $p$ and $q$ such that the segment $q-p$ is aligned perpendicular to some axis of symmetry passing through $v$ and $q$ is closer to this axis then $p$.

See Figure (7) for a illustration of directions of the axis-monotonicity and $D_{4}$ symmetry.

Lemma 31. [3] If the starting (possibly unstable) configuration has $D_{4}$ symmetry and is axis-monotone, then so is the function $f: \mathbb{Z}^{2} \rightarrow \mathbb{N}$, where $f(v)$ is the number of times the site $v$ topples. 


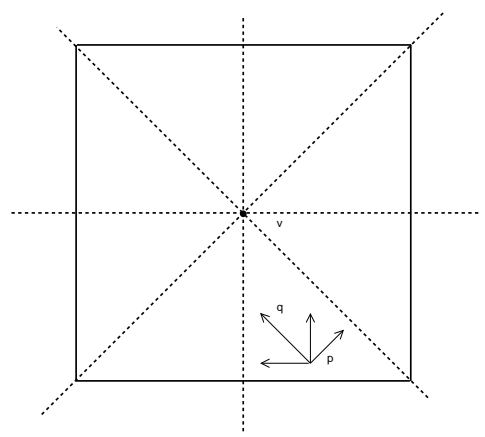

Figure 7: Axis Monotonicity of a function

In the present context, the function of interest will be the number of particles present at a particular node. We observe that any function which is $D_{4}$ symmetric, axis-monotone, and has finite support, is sandwiched inside an $l_{1}$ ball (diamond) and $l_{\infty}$ ball (square) of same radius. The number of sites contained in both these balls is quadratic in the radius. To be precise, denoting an $l_{\infty}$ ball of radius $n$ around $v$ by $B_{\infty}(v, n)$, the volume of this ball is the maximum of particles it can hold in any stable configuration. This is given by

$$
\left|B_{\infty}(v, n)\right|=3(2 n+1)^{2} .
$$

Similarly, the maximum weight of any stable configuration on $B_{\infty}(v, n)$ is bounded by the following:

$$
\left|B_{1}(v, n)\right|=6 n^{2}+6 n+3
$$

The balls, therefore can hold at most $\Theta\left(n^{2}\right)$ particles. When one starts adding particles at node $v$, clearly adding $\left|B_{1}(v, n)\right|=\Theta\left(n^{2}\right)$ particles is enough to flood an $l_{1}$ ball of radius $n$ around $v$. The property (hLC) follows immediately.

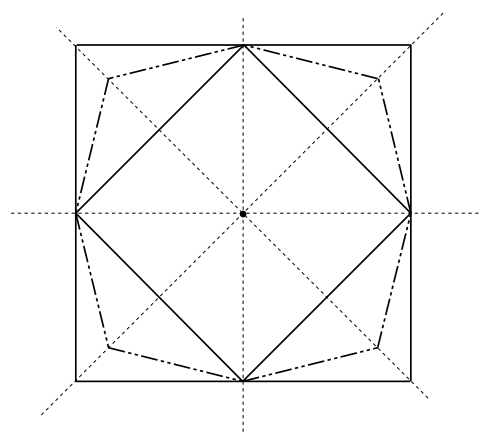

Figure 8: A example of a function support which is $D_{4}$-symmetric and axis monotone (about the center of the grid). The function support is shown in broken lines and bold lines depict the $l_{\infty}$ and $l_{1}$ balls

The property (LS) requires more work. Here we start with placing some $h$ particles at each node in $B_{1}(v, r)$. This configuration is axis-monotone and $D_{4}$-symmetric. 
Consequently, the stable configuration resulting from this is also axis-monotone and $D_{4^{-}}$ symmetric. Let $h$ be smallest such number such that the resulting configuration contains the ball $B_{1}(v, R)$. The total number of particles this configuration can have is $\Theta\left(R^{2}\right)$. The particles we started of with are $h \Theta\left(r^{2}\right)$. Hence, $h$ is $\Theta\left((R / r)^{2}\right)$. The property (LS) follows.

\section{$7 \quad$ Future work}

In the present work, we generalize the approach taken by Babai and Gorodezky [3]. The analysis raises lot of interesting questions, answers to which will aid our understanding of random walks and sandpiles. We refer the reader to a slightly expanded version of this paper [12] which has additional expository sections describing, in limited details, the parallels with classical complex analysis and the intuitive connections between measures induced by random walks on graphs, and Brownian motion in Euclidean spaces and as implications of these, informally argue that the graph properties we have derived are analogous to essential properties of Euclidean spaces which allow efficient diffusions.

In the context of the transience class problem, the central question is that pertaining to a complete characterization of graph properties which are equivalent to polynomial bounds on tcl. The present result shows that under some very general and reasonable assumptions, the polynomial bounds can be established. There are some obvious extensions possible from our theorems. For example if one considers graphs made from attaching two graphs each individually satisfying the required preconditions but having different volume growth parameters. One can bound the Harnack's constant for both components separately and bound the one for the whole graph using the simple triangle inequality. But apart from these trivial modifications, we do not yet know of any class of sandpile which are not covered by our result. Supported by the analogy with the continuous case, we believe the assumptions we make are essential and cannot be substantially relaxed. A related sub-question is finding non-lattice type graphs satisfying the properties we mention.

The second question supplements the first one. Consider any Euclidean space and triangulate it using simplices or cubic complexes, basically any regular tessellation will do. We are interested in the graph of this triangulation complex. Indeed, the grid sandpile are obtained in exactly this manner from the two dimensional Euclidean space. In this graph, consider a subgraph corresponding to any open set. All edges leading to points outside the set are connected to the sink node. In this setting, one can see that polynomial bounds in sandpile settings roughly correspond to non-trivial bounds on the Harnack's constant in the continuous space. Any lower dimensional set in $\mathbb{R}^{n}$ has unbounded Harnack's constant. For some simple cases, it can be shown that the sandpile corresponding to these sets also have transience classes which cannot be polynomially bounded. Babai and Gorodezky [3] first mention this for the line sandpile. The case of general degenerate sets is similar. The only crucial component is the fact that from every node, there is an edge to sink node. One can see an interesting confluence of the notion of bad models here. In the setting of probability spaces with the Lebasgue-like measure, full dimensional sets are used for modeling events with non-zero probability, while the lower-dimensional 
ones are considered beneath consideration and encapsulate unlikely events. On similar lines, Babai and Toumpakari [4], and Babai and Gorodezky [3] make case for the notion of polynomially bound transience classes as the essential qualification for a sandpile to be a good model. Dhar [20] mentions several physical phenomena which sandpiles are intended to model. In all these settings, the sandpile graphs arise as tessellation graphs of the underlying continuous space. It is only natural to expect that if the underlying set itself is a bad model, the sandpile on top of it is going to a bad model. This leads us to a question impinging on the notion of dimensions for graphs. For the family of lattice graphs based sandpiles, does every bad sandpile correspond to a degenerate set?

To conclude, we bring together ideas from three different areas of mathematics. Our bounds demonstrate that the research in each of these areas has ramifications in the other two. This magnifies the importance of resolving any question in any of these areas. We hope that this paper motivates further investigation on sandpile-like discrete diffusions, in analogy with random walks and complex analysis.

\section{References}

[1] S. Arora, S. Rao and U. V. Vazirani. Expander flows, geometric embeddings and graph partitioning. Proceedings of the 36th Annual ACM Symposium on Theory of Computing, 222-231, 2004.

[2] Personal communication with Lazlo Babai.

[3] L. Babai and I. Gorodezky. Sandpile transience on the grid is polynomially bounded. Proceedings of the 18th ACM-SIAM symposium on Discrete algorithms, 627-636, 2007.

[4] L. Babai and E. Toumpakari. A Structure Theory of the Sandpile Monoid for Directed Graphs. to appear in the Journal of Combinatorics. (A preliminary version appears in Chapters 1-4 of E. Toumpakari's dissertation, "On the Abelian Sandpile Model," University of Chicago 2005. See http://people.cs.uchicago.edu/ laci/students/ )

[5] K. Ball. An Elementary Introduction to Modern Convex Geometry. Flavors of Geometry (edt. Silvio Levy), MSRI, 31, 1997.

[6] I. Benjamini and L. Lovász. Harmonic and Analytic functions on Graphs. Journal of Geometry, 76(1-2):3-15, 2003.

[7] N. Biggs. Chip-Firing and the Critical Group of a Graph. Journal of Algebraic Combinatorics, 9:25-45, 1999.

[8] A. Bjorner, L. Lovasz and P. W. Shor. Chip-firing games on graphs. European Journal of Combinatorics, 12:283-291, 1991.

[9] B. Bollobás. Modern Graph Theory. Graduate Texts in Mathematics, SpringerVerlag, 184.

[10] A. Choure and S. Vishwanathan. Random Walks, Electric Networks and The Transience Class problem of Sandpiles. Preprint, 2011. arXiv:1105.3368 
[11] A. Choure and S. Vishwanathan. Random Walks, Electric Networks and The Transience Class problem of Sandpiles. Proceedings of the 23rd annual ACM-SIAM symposium on Discrete algorithms, 1593-1611, 2012.

[12] A. Choure and S. Vishwanathan. On graph parameters guaranteeing fast Sandpile diffusion. Preprint, 2012. arXiv:1207.0421

[13] P. Christiano, J. Kelner, A. Madry, D. Spielman and S. Teng. Electrical Flows, Laplacian Systems, and Faster Approximation of Maximum Flow in Undirected Graphs. Proceedings of the 43rd ACM symposium on Theory of computing, 273-282, 2011.

[14] F. R. K. Chung and S.-T. Yau. A Harnack inequality for homogeneous graphs and subgraphs. Communications on Analysis and Geometry, 2:627-640, 1994.

[15] F. R. K. Chung and S.-T. Yau. Eigenvalues of graphs and Sobolev inequalities. Combinatorics, Probability and Computing, 4:11-26, 1995.

[16] F. R. K. Chung and S.-T. Yau. Logarithmic Harnack inequalities. Mathematical Research Letters, 3:793-812, 1996.

[17] F. R. K. Chung and S.-T. Yau. A Harnack inequality for Dirichlet eigenvalues. Journal of Graph Theory, 34:247-257, 2000.

[18] R. Cori and D. Rossin. On the Sandpile Group of Dual Graphs. European Journal of Combinatorics, 21:447-459, 2000.

[19] T. Delmotte. Harnack inequalities on graphs. Séminaire de Théorie spectrale et géométrie, 16:217-228, 1997-1998.

[20] D. Dhar. Theoretical studies of self-organized criticality. Physica A: Statistical and Theoretical Physics, 369(1):29-70, 2006.

[21] D. Dhar. Self-organised critical state of sandpile automaton models. Phys. Rev.Lett., 64:1613-1616, 1990.

[22] D. Dhar, P. Ruelle, S. Sen and D. Verma. Algebraic aspects of abelian sandpile models. Jour. Phys. A, 28:805-831, 1995.

[23] P. Diaconis and L. Saloff-Coste. Logarithmic Sobolev Inequalities for Finite Markov Chains. The Annals of Applied Probability, 6(3):695-750, 1996.

[24] P.G. Doyle and J.L. Snell. Random walks and electric networks. The Carus Math. Monographs, Math. Association of America, 22, 1984.

[25] S. Hoory, N. Linial and A. Wigderson. Expander graphs and their applications. Bulletin of AMS, 43:439-561. 2006.

[26] Y. Hou, T. Lei and C. Woo. On the sandpile group of the graph $K_{3} \times C_{n}$. Linear Algebra and its Applications, 428:1886-1898, 2008.

[27] Y. Hou, C. Woo and P. Chen. On the sandpile group of the square cycle $C_{n}^{2}$. Linear Algebra and its Applications, 418:457-467, 2006.

[28] D. Jerison, L. Levine and S. Sheffield. Internal DLA in Higher Dimensions. Preprint, 2010. arXiv: 1012.3453 
[29] J. Kelner and A. Madry. Faster Generation of Random Spanning Trees. Proceedings of the 50th Annual IEEE Symposium on Foundations of Computer Science, 13-21, 2009.

[30] M. Kleber. Goldberg Variations. Math. Intelligencer, 27(1):55-63, 2005.

[31] I. Koutis, G. L. Miller and R. Peng. Approaching Optimality for Solving SDD Linear Systems. Proceedings of the 51st Annual IEEE Symposium on Foundations of Computer Science, 235-244, 2010.

[32] I. Koutis, G. L. Miller and R. Peng. A Nearly- $m \log n$ Time Solver for SDD Linear Systems. Proceedings of the 52nd Annual IEEE Symposium on Foundations of Computer Science, 590-598, 2011.

[33] G. F. Lawler and V. Limic. Random Walk: A Modern Introduction. Cambridge Studies in Advanced Mathematics, 123, 2010.

[34] L. Levine and Y. Peres. Strong spherical asymptotics for rotor-router aggregation and the divisible sandpile. Potential Analysis, 30:1-27, 2009.

[35] L. Levine and Y. Peres. Scaling limits for internal aggregation models with multiple sources. J. Anal. Math., 111:151-219, 2010.

[36] L. Lovász. Random walks on graphs: A survey. Combinatorics, Paul Erdös is Eighty, János Bolyai Math. Soc., 353-397, 1993.

[37] C. St. J. A. Nash-Williams. Random walk and electric currents in networks. Mathematical Proceedings of the Cambridge Philosophical Society, 55:181-194, 1959.

[38] P. Mörters and Y. Peres. Brownian Motion. Cambridge University Press, 2010.

[39] P. Miltersen. The Computational Complexity of One-Dimensional Sandpiles. Theory of Computing Systems, 41:119-125, 2007.

[40] W. Rudin. Real and Complex Analysis, International Series in Pure and Applied Mathematics, McGraw-Hill, $3^{\text {rd }}$ ed., 1986.

[41] M. Schulz. An NP-complete Problem for the Abelian Sandpile Model. Complex Systems, 17:17-28, 2007.

[42] J. Shen and Y. Hou. On the sandpile group of $3 \times n$ twisted bracelets. Linear Algebra and its Applications, 429:1894-1904, 2008.

[43] D. Spielman and N. Srivastava. Graph sparsification by effective resistances. Proceedings of the 40th Annual ACM Symposium on Theory of Computing, 563-568, 2008.

[44] D. A. Spielman and S. Teng. Nearly-Linear Time Algorithms for Preconditioning and Solving Symmetric, Diagonally Dominant Linear Systems. http://arxiv.org/abs/cs/0607105.

[45] G. Tardos. Polynomial bound for a chip firing game on graphs. SIAM Journal of Discrete Mathematics, 1:397-398, 1988.

[46] A. Telcs. The Art of Random Walks, Lecture Notes in Mathematics, 1885, 2006. 
[47] A. Telcs. Random walk on graphs with regular resistance and volume growth. Ann. Inst. H. Poincaré Probab. Statist., 44(1):143-169, 2008.

[48] E. Toumpakari. On the sandpile group of regular trees. European Journal of Combinatorics, 28:822-842, 2007.

\section{A Basic Potential Theory}

For a proper introduction to harmonic functions on graphs, we refer the reader to the beautiful paper by Benjamini and Lovasz [6]. See Telcs [46] for a thorough view. We start with some important definitions and fundamental properties. Given a connected graph $G$ and a function $\pi: V(G) \rightarrow \mathbb{R}$, we say that $\pi$ is harmonic over the vertex set $V_{h}$ if

$$
\frac{1}{\operatorname{degree}(v)} \sum_{u \sim v} \pi(u)=\pi(v) \quad v \in V_{h} .
$$

The vertices in $V-V_{h}$, adjacent to any vertex in $V_{h}$, are called the "poles" of $\pi$. The set $V_{h}$ is also called the interior of $\pi$ and the set of poles referred to as the boundary. Being harmonic over $V_{h}$ means that the value of $\pi$ at any vertex in $V_{h}$ is the average of its value in the immediate neighborhood. In case of multi graphs, we take the appropriate weighted means, where the weights are the number of common edges. This leads us to the first basic property,

Property 32. Any non-constant harmonic function can assume its extreme values only at the set of poles.

It follows that every non-constant harmonic function has at least two poles, its maxima and minima. Such functions are completely determined by their values on these vertices. Formally speaking,

Property 33. Uniqueness: If two functions harmonic on $V_{h}$ agree on the boundary, they agree everywhere in the interior.

Property 33 is important as it allows one considerable freedom in constructing harmonic completions of functions defined over the boundary set. This problem is the discrete analogue of the classical boundary value problems in complex analysis. If one fixes the interior set $V_{h}$ and allows arbitrary boundary values, the complete set of harmonic functions is obtained. This set is closed under linear combinations and contains all the constant functions.

Property 34. The set of functions harmonic over any set $V_{h}$ form a vector space.

Two important scenarios in which these functions arise naturally are electric networks and random walks.

Electric Networks: Consider a resistive electric network (i.e. a circuit made up entirely of resistors). Let ${ }_{s} \pi_{t}(v)$ be the potential that appears at node $v$ when unit potential is 
applied across $t$ and $s$. Using the equation of charge conservation (Kirchoff's node law), one can show that these potentials are harmonic on all nodes except $s$ and $t$.

Random Walks on Graphs-dipole version: Consider a graph $G$ and two special vertices $s$ and $t$. The potential associated with $v$, with $s$ and $t$ as poles, ${ }_{s} \pi_{t}(v)$ is defined as the probability of reaching $t$ before $s$ starting from $v$. One can check that the function $\pi$ so defined is indeed harmonic on the set $V-\{s, t\}$, with the maximum value of 1 at the node $t$ and the minimum value 0 at $s$. The generalization to the multi-pole situation is simple, but it is interesting for a different reason.

Random Walks on Graphs- multipole version: Again we consider graph $G$, but this time we have a set of interior nodes $V_{h}$ and the corresponding set of poles, $P=\left\{s_{i}\right\}$. Denote by $\pi_{j}(v)$, the probability of a random walk, starting at $v$, hitting $s_{j}$ before any other vertex in $S$. Assign any desired set of values $\pi\left(s_{i}\right)$ to each of these poles. The value $\pi(v)$ is defined as the sum $\sum_{j} \pi\left(s_{j}\right) \pi_{j}(v)$. As before, it is a simple exercise to check the function $\pi$ is harmonic over $V_{h}$. The formula basically suggests taking an expectation over the boundary values, using the measure induced by the random walk.

The main implication here is that one can intuitively think of the electric network theory as an analysis of random walks of electrons on the underlying graphs. Consequently, results from network theory can be used to prove interesting facts in other related areas. As an example, consider the problem of constructing the harmonic completion of a function with given boundary values. All one needs to do is to take the corresponding circuit and apply potentials equal to the boundary values on the boundary points. The potentials that will appear on other nodes can be computed using basic linear algebra (the only non-trivial step involves inverting the combinatorial Laplacian of $G$ ) thus allowing construction of harmonic completions efficiently.

The reciprocity theorem can be restated in terms of just potential sources and potential measurements using the notion of effective resistances between pairs of nodes. The effective resistance between a pair of nodes $u$ and $v, R_{e f f}(u, v)$ is defined as the potential difference which develops between $u$ and $v$ if a unit current source is applied across $u$ and $v$. In any resistive network, the following reciprocity property holds. See [10] for an elementary proof.

Lemma 35. Potential Reciprocity Lemma : If taking $s$ and $t$ as poles with $\pi(s)=0$ and $\pi(t)=1$ induces a potential of ${ }_{s} \pi_{t}(v)$ at node $v$ and interchanging the roles of $v$ and $t$ induces ${ }_{s} \pi_{v}(t)$ at $t$ then,

$$
R_{e f f}(s, t)_{s} \pi_{t}(v)=R_{e f f}(s, v)_{s} \pi_{v}(t) .
$$

In particular, when the effective resistances across $s$ and $t$ are the same as $s$ and $v$, we have ${ }_{s} \pi_{t}(v)={ }_{s} \pi_{v}(t)$. In the following discussion, we will omit the left subscript $(s)$ from ${ }_{s} \pi_{t}$ whenever it is clear from context. We say that a walk $P$ is an instance of $\pi_{s}$ if it starts at some vertex $v$, avoids $s$ and ends at $t$. The following lemma may already be known to experts. A proof appears in [10].

Lemma 36. A triangle inequality for potentials:

$$
\pi_{i}(j) \pi_{j}(k) \leqslant \pi_{i}(k) .
$$




\section{B Volume properties of lattice graphs}

Consider a lattice in $\mathbb{Z}^{k}$ and the resulting graph, where each lattice point is in the neighborhood of the $2 k$ points flanking it. In this graph, consider a ball $\mathbf{B}_{r}$, and the annulus $\mathbf{B}_{r-c, r}$. [5] notes the volume of an $L_{1}$ ball as,

$$
\left|\mathbf{B}_{r}\right|=\frac{(2 r)^{k}}{k !}
$$

The simpler cases with dimensions 2 , and 3 are easy to directly visualise. The higher dimension version follows simply from induction. Number of nodes in $\mathbf{B}_{r}$ is approximable as follows.

$$
\#_{V}\left(\mathbf{B}_{r}\right)=\frac{(2 r)^{k}}{k !}+o\left(r^{k}\right)
$$

In the above expression, the second term on right hand side is a lower order correction factor which compensates for the discrepancy due to points on the surface of the convex ball under consideration. The constants in this lower order term may be functions of dimension. Using equation (18), the volume of annular regions can be approximated as follows.

$$
\begin{aligned}
\left|\mathbf{B}_{r-c, r}\right| & =\frac{(2 r)^{k}}{k !}-\frac{(2(r-c))^{k}}{k !} \\
& =\frac{(2 r)^{k}}{k !}(1-c / r)^{k}
\end{aligned}
$$

Using equations (18),(20), the proportion of complete sphere's volume contained in the annular region can be seen as

$$
\frac{\left|\mathbf{B}_{r-c, r}\right|}{\left|\mathbf{B}_{r}\right|}=(1-c / r)^{k}
$$

For all values of $r>c$, we have the trivial inequality,

$$
\left|\mathbf{B}_{r-c, r}\right| \leqslant\left|\mathbf{B}_{r}\right|
$$

Imposing the constraint $r>2 c$, we obtain the reverse version,

$$
\left|\mathbf{B}_{r}\right| \leqslant 2^{k}\left|\mathbf{B}_{r-c, r}\right|
$$

The number of lattice points in these regions follow identical ratio bounds with lower order correction factors which asymptotically go to zero. We therefore have the following asymptotic vertex count bounds.

$$
\#_{V}\left(\mathbf{B}_{r-c, r}\right) \leqslant \#_{V}\left(\mathbf{B}_{r}\right) \leqslant 2^{k} \#_{V}\left(\mathbf{B}_{r-c, r}\right) \quad \forall r \geqslant 2 c
$$

THE EleCtronic Journal of COMBinatorics 22(1) (2015), \#P1.44 
We will be needing the ratio of the volumes of two balls. Consider the balls $\mathbf{B}_{r-c}$, and $\mathbf{B}_{r}$ (using $L_{1}$ metric).

$$
\frac{\left|\mathbf{B}_{r-c}\right|}{\left|\mathbf{B}_{r}\right|}=(1-c / r)^{k}
$$

For values of $r$ satisfying the constraint $r>2 c$, this basically translates to the following bound.

$$
\left|\mathbf{B}_{r}\right| \leqslant 2^{k}\left|\mathbf{B}_{r-c}\right|
$$

Again, barring lower order correction factors, the following asymptotic result holds.

$$
\#_{V}\left(\mathbf{B}_{r}\right) \leqslant 2^{k} \#_{V}\left(\mathbf{B}_{r-c}\right) \quad \forall r \geqslant 2 c
$$

